Covered in: Web of Sciences (WOS); EBSCO; ERIH+; Google Scholar; Index Copernicus; Ideas RePeC; Econpapers; Socionet; CEEOL; Ulrich ProQuest; Cabell, Journalseek; Scipio; Philpapers; SHERPA/RoMEO repositories; KVK; WorldCat; CrossRef; CrossCheck

2018, Volume 9, Issue 3, pages: 119-142 | doi: https://doi.org/10.18662/po/39

\section{Intensionality, Reference and Games of Partial Information II. Semantic Verbs}

\section{Mihai HîNCU1}

\footnotetext{
1 Teaching Assistant, Faculty of Political Sciences, Letters and Communication, Valahia University of Târgovişte, Târgovişte, Romania, mihaihincu@gmail.com
}

\begin{abstract}
In this paper, I look at the semantic behaviour of the most emblematic verb of our semantic lexicon, „refer (to)", through the prism of the semantic features considered definitory for the extensional verbs and I show that there are strong theoretical reasons for considering that it belongs to the class of ITVs and that, in consequence, it generates structural ambiguity at each level of language in which it is involved. In this regard, I show how the conceptual framework of the games of partial information can successfully accommodate the semantic verb's inherent ambiguity. In order to accomplish this task, I present a scenario of strategic communication involving semantic verbs and I model it as a two-agent coordination game. The cognitive dynamics peculiar to the agents' interaction will be presented on the background of some reasonable assumptions introduced to guarantee the finding of a solution concept corresponding to the game. In this regard, after I compute the expected utilities of the strategic profiles, I determine a set of Nash equilibria and I show that the game's unique solution can be equated with that set's member which passes the test of Paretian efficiency. In the end, I present the picture of the interplay between the speaker's reference and the semantic reference which emerges from the game-theoretical framework adopted here and I show some of its methodological consequences related to the way in which the key concept of reference has to be theoretically framed. This is the second part of a two-part study.
\end{abstract}

Keywords: Exportation; monotonicity; speaker's reference; interpretation de dicto; Nash equilibrium; Pareto efficiency; intensionality.

How to cite: Hîncu, M. (2018). Intensionality, Reference and Games of Partial Information II. Semantic Verbs. Postmodern Openings, 9(3), 119-142. https://doi.org/10.18662/po/39 


\section{Introduction}

In the first part of the present paper, I scrutinized the semantic differences between the ITVs and the extensional transitive verbs with respect to the existential entailment, to the substitution salva veritate of the coextensional terms and to the semantic specificity. Seen through the prism of the semantic features considered definitory for classifying a transitive verb as being extensional, the semantic behaviour of the verbs from our semantic lexicon significantly resembles that of the ITVs'.

In this part I will show that there are strong theoretical reasons for considering that the verbs making up the semantic lexicon belong to the class of the ITVs and that, in consequence, they too are ambiguous between an intensional, de dicto interpretation and an extensional, de re interpretation. In this regard, I choose to exclusively orient my presentation of the semantic verbs' intensional profile and my game-theoretical analysis which I'll offer in the third section, towards the most emblematic verb of our semantic idiom, the semantic verb ,refer (to)".

As far as I know, the only author who endorsed the thesis that the semantic verbs are ITVs, and who in this regard offered, in (D'Ambrosio, 2017) robust empirical evidence supporting this thesis, is Justin D'Ambrosio, even though some recent theoretical considerations about the intensionality of the most representative semantic verb mentioned above can also be found in (Sainsbury, 2018). After I present, in the next section, the theoretical reasons favouring the view that the standard semantic verb , refer (to)" is an ITV, I will end it by reviewing the empirical data regarding the verb's intensionality offered by D'Ambrosio in (D'Ambrosio, 2017).

In the third section, I present a scenario of strategic communication in which the above semantic verb is involved and I show how its inherent ambiguity at the utterance level is rationally solved in a two-agent game of partial information by means of which I model this particular communication situation. After I computationally identify the Pareto efficient Nash equilibrium which constitutes the game's unique solution, I will show, in the fourth section, which picture of the interplay between the speaker's reference and the semantic reference emerges from the conceptual framework of the games of partial information.

\section{The Intensionality of Semantic Verbs}

In daily conversations, the verb „refer (to)” can be used to designate people existing in the actual world, like Woody Allen. In case Stephanie is 
talking, in a context, about Woody Allen, the following sentence will be a true one:

(1) Stephanie is referring to Woody Allen.

Insofar as the person denoted by the proper name which occupies the object position of the verb in (1) exists, it is obvious that, in this case, the intensional complement has ontological import and, in consequence, it inferentially generates an existential entailment. But the semantic verb from (1) can also occur in sentences whose readings lack existential import, like:

(2) Stephanie is referring to a round square.

In case there is a context in which Stephanie is referring verbatim to a round square, the sentence (2) is true in that context even though the referent of the indefinite NP-complement fails to exist in the real world. In this scenario, the existence of the entity mentioned by the linguistic material from the intensional verb construction, is alethically irrelevant. To the extent that both the semantic verbs, like ,refer (to)", and the ITVs are accompanied by NP-complements lacking existential import, and that the sentences in which these verbs are embedded admit readings blocking the inferential pattern of existential entailment, it can be concluded that both types of verbs display the same semantic behaviour, and that the verbs of our semantic lexicon are intensional transitives (D'Ambrosio, 2017; Moltmann, 1997; Sainsbury, 2018).

If the semantic verb „refer (to)” is an intensional one, a sentence in which it occurs will allow interpretations with different truth-conditional profiles (D'Ambrosio, 2017). Consider the following sentence:

(3) Stephanie is referring to a philosopher.

The sentence (3) admits an interpretation on which it is true just in case the NP-complement of the semantic verb has a particular satisfier. That (3) licenses an interpretation of this kind is confirmed by the fact that the verbal construction which occurs in (3) can be successfully accompanied by an expression which makes explicit, in the surface syntax, the requirement of a specific satisfier for the indefinite NP, as the following example shows:

(4) Stephanie is referring to a particular philosopher. 
But the sentence (3) can receive a second interpretation on which it is true even though the NP-complement of the semantic verb does not have one specific satisfier (D'Ambrosio, 2017; Sainsbury, 2018). In this sense, in case the extension of the indefinite which occurs in the sentence (3) is not empty, and in consequence, there are multiple satisfiers, the truth of (3) is compatible with all of them. On this latter interpretation of the sentence (3), in contrast with its former interpretation, highlighted in (4), the specificity condition is violated. This fact too is well supported by linguistic evidence (D'Ambrosio, 2017; Forbes, 2013; Moltmann, 1997). In this regard, the semantic verb which occurs in (3) tolerates expressions which semantically trigger non-specificity and, in consequence, it makes a sentence, like the following one, to be semantically well-behaved:

(5) Stephanie is referring to a philosopher, but not to a particular one.

Insofar as the sentences comprising semantic verbs resemble the sentences consisting of ITVs, in that they are both open to interpretations with different truth-conditional profiles, it can be said that the former sentences are similarly ambiguous (D'Ambrosio, 2017). With regard to the sentence (3) displayed above, this means that (3) too is ambiguous between two different scope interpretations of the linguistic material which occupies the verb's object position with regard to the semantic verb which occurs in (3) (Lappin, 2017). Moreover, the explanation of its ambiguity appeals to the very same logical machinery of scope mobilized to explain the structural ambiguity of the sentences with intensional verbs between a de re, extensional interpretation, and a de dicto, intensional interpretation (D’Ambrosio, 2017; Forbes, 2013; Moltmann, 1997; Recanati, 2000; Richard, 2013).

On the de re interpretation of the sentence (3), the semantic verb takes narrow scope and its interpretation is logically controlled by the interpretation of the quantified complement which, given its wide scope with respect to the verb, forces a reading according to which the NP has a particular satisfier, more precisely, a reading on which there is a particular philosopher to which the referent of the subject phrase of (3) is referring to.

In contrast, on the de dicto interpretation of the sentence (3), the semantic verb takes wide scope over the indefinite NP-complement and logically constrains it, at the level of interpretation, to the effect that the indefinite now receives a non-specific reading, and in consequence, is compatible with many satisfiers, in case there are such entities. More 
precisely, on the de dicto interpretation of the sentence (3), highlighted by the semantically equivalent sentence (5), the referent of the subject phrase of (3) is not referring to a particular philosopher, insofar as many of the complement's satisfiers are eligible for the semantic job of referring.

Usually, it is considered that on the de dicto interpretation of a sentence with ITV, in contrast with what happens on its de re interpretation, the NP-complement of the intensional verb cannot be exported from the verb's object position to the subject position (Almog, 1998; Forbes, 2013; Moltmann, 1997; Recanati, 2000; Richard, 2013; Sainsbury, 2018). Even though this logical claim is also validated by the de dicto interpretations of the sentences in which semantic verbs occur, like (3) above, some clarification is required in order to get a better grasp of the issue. The exportation operation comes in two varieties, a strong one and a weak one (Sainsbury, 2018). In case the NP-complement of the semantic verb from the sentence (3) is subjected to the weak exportation operation, the following deductive inference is valid on the sentence's de dicto interpretation (Moltmann, 1997; Sainsbury, 2018):

(WE): Stephanie is referring to a philosopher. There is something Stephanie is referring to.

On the very same interpretation of the sentence (3), insofar as the set of the indefinite NP's satisfiers can be empty or it can contain unspecific satisfiers, the exportation from the premise (3) is not allowed (Richard, 2013; Sainsbury, 2018) and, in consequence, the following deductive inference, which corresponds to the strong exportation operation, is not valid:

(SE): Stephanie is referring to a philosopher.

There is some philosopher Stephanie is referring to.

In the opposite direction, the interpretation which the sentences with semantic verbs or ITVs have in common with the sentences consisting of extensional transitive verbs, namely the de re interpretation, validates the strong exportation from (3). On this interpretation of (3), insofar as the set of satisfiers required by its NP-complement is a singleton, a fortiori the weak form of exportation operation is permitted.

Another aspect in which the semantic verb „refer (to)” is similar to an ITV is best highlighted when the former's semantic behaviour regarding the substitution of extensionally equivalent terms is taken into consideration (D'Ambrosio, 2017; Sainsbury, 2018). As the previous part of the present 
study has shown, a sentence which contains an intensional verbal construction can be interpreted in two ways. On its extensional, de re interpretation, the sentence obeys the inferential pattern of substitution, while on its intensional, de dicto interpretation the substitution salva veritate of coreferential expressions fails (Abbott, 2010; Almog, 1998; Forbes, 2013; Moltmann, 1997; Recanati, 2000). In case the paradigmatic semantic verb „refer (to)" would be an intensional verb, it would allow too distinct interpretations which would elicit different semantic responses to the substitution pattern. Unsurprisingly, the linguistic data support this prediction (D'Ambrosio, 2017). In order to illustrate, let us consider the following sentence:

(6) Stephanie is referring to Pablo Neruda.

If there is, in the actual world, a situation in which the referent of the subject phrase from (6) is referring to the referent of the proper name which occupies, in the same sentence, the verb's object position, that situation grounds the truth of (6). On the extensional, de re interpretation of the above sentence, the semantic verb is referentially transparent in object position. In this sense, as the complement of the semantic verb from (6) licenses the substitution salva veritate of a coreferential expression, like the proper name „Neftali Reyes Basoalto”, the following sentence is also true:

(7) Stephanie is referring to Neftali Reyes Basoalto.

There is also another interpretation of the sentence (6) on which the semantic verb linguistically profiled in it, is not referentially transparent in object position. According to this reading which induces referential opacity, the substitution of the poet's real name for his pen name, in the intensional VP of (6), does not support the truth of the sentence (7). If, for example, the sentence (6) is used, in a context, to make a report, and that context makes salient that the referent of the subject phrase from (6) lacks information about the coreferentiality of the already mentioned two proper names, the substitution salva veritate of these extensionally equivalent expressions, in the verb's complement of (6), is not a valid operation.

Another logical trait which the semantic verbs have in common with the majority of intensional verbs is emphasized by the assessement of how well the former's semantic behaviour responds to monotonicity (D’Ambrosio, 2017; Sainsbury, 2018; Zimmermann, 1993, 2006). In this regard, the monotonicity of the semantic verb ,refer (to)" is attested by the 
fact that the inferential pattern displayed below has valid instances when the emblematic verb from our semantic idiom is interpolated between the subject phrase and the object phrase:

$$
\begin{array}{ll}
\text { Monotonicity: } & \mathbf{S ~ V ~ \mathbf { O } ^ { 1 }} \\
& \frac{\mathbf{O}^{1} \rightarrow \mathbf{O}^{2}}{\mathbf{S ~ V ~ \mathbf { O } ^ { 2 }}}
\end{array}
$$

In this sense, the following inference, in which the semantic verb is present both at level of premise and at the level of conclusion, and in which the extension of the indefinite NP-complement from the premise is a subset of the extension of the indefinite NP-complement mentioned in the conclusion, is valid on both the de dicto and the de re interpretation of the semantic verb when its reading is invariant across the sentences:

\section{$\left(\mathrm{M}_{1}\right)$ : $\quad$ Stephanie is referring to a yellow poppy. Stephanie is referring to a poppy.}

Likewise, the presence of the negation operator in the above sentences' surface syntax reverses the inferential pattern and confirms the monotonicity of the semantic verb by making valid the following deduction:

\section{$\left(\mathrm{M}_{2}\right)$ : Stephanie is not referring to a poppy. \\ Stephanie is not referring to a yellow poppy.}

Besides the above theoretical reasons supporting the claim that the semantic verb ,refer (to)" is an ITV, there is also empirical support in favour of its confirmation. In this regard, D'Ambrosio offered in (D'Ambrosio, 2017) robust empirical evidence which shows that the most important verb from our semantic lexicon exhibits intensionality and, in consequence, it semantically behaves like an ITV. In his groundbreaking paper, he designed and conducted three experiments, contrasting the semantic verb with a paradigmatically intensional verb and with a paradigmatically extensional verb, and he measured the statistical differences between the transitive verbs' semantic behaviours with respect to the existential entailment, to the substitution salva veritate of the coreferential terms and to the semantic specificity. Bear in mind that this is the only empirical investigation to date.

The collected data from the first experiment testing the way in which the target verbs, to which the subjects were exposed, elicit existential entailment, show that the participants align the semantic verb more closely 
to the ITVs than to the extensional verbs. In this regard, on a Likert scale from 1 to 7, in which the low score indicated that a verb's reading allows existential import, while the high score indicated that the existential entailment is inferentially blocked, the mean rating of the semantic verb (M $=4.99)$ was closer to that of the ITVs $(\mathrm{M}=5.35)$ and it significantly surpassed the mean rating of the extensional verbs $(\mathrm{M}=1.96)$. The second experiment conducted by D'Ambrosio, which tested whether the semantic verb licenses intensional, de dicto readings, also found that the subjects semantically place this verb in the proximity of the ITVs. Thus, on a 7 point Likert scale, in which the low score indicated that a verb does not license non-specific de dicto readings and the high score indicated the opposite, the ratings of the semantic verb $(\mathrm{M}=5.14)$ were significantly closer to the ratings of the ITVs $(\mathrm{M}=5.99)$ and outclassed those of the extensional verbs $(\mathrm{M}=3.45)$. Finally, D'Ambrosio conducted an experiment which tested how the most representative verb of our semantic lexicon behaves with respect to the substitution salva veritate of the coextensional terms. In this regard, on a Likert scale from 1 to 7 , in which the low score indicated, this time, that a verb allows interpretations in which the substitution of the semantically equivalent terms does not preserve the initial truth-value of a sentence, the mean rating of the semantic verb $(M=4.19)$ was closer to that of the ITVs $(\mathrm{M}=3.61)$ and it significantly differed from the mean rating of the extensional verbs $(M=5.65)$. The overall pattern of data collected from all these three empirical investigations presented in (D'Ambrosio, 2017) confirm, once again, that the emblematic verb from our semantic idiom is an ITV, and so, that it semantically displays ambiguity.

\section{Reference Games}

In this section, using the conceptual framework of games of partial information presented in (Clark, 2012; Parikh, 1991, 2000, 2001, 2007, 2010), I will show how the strategic interaction involved in the communication between a speaker, who chooses to utter, in a particular context, a sentence in which occurs a semantic verb, and a hearer who has to choose, in order to resolve the ambiguity inherent to the intensional verb construction, between an intensional, de dicto and an extensional, de re interpretation, can be modeled as a two-agent game whose unique solution concept corresponds to a Pareto-efficient Nash equilibrium. In this regard, as I said in the first section, I choose to exclusively orient my gametheoretical analysis of the semantic verbs towards the emblematic verb 
„refer (to)" which, as we have seen in the previous section, belongs to the class of ITVs.

Let us look at the following scenario. Three old friends, Mikhael, Daniel and Stephanie, have to meet one day in a restaurant. Mikhael, who is fascinated with linguistic stuff, recently told Stephanie the story about how the ghost word „dord" has occurred in the 1934 edition of the Webster's Dictionary as a result of someone misreading a note, submitted by the chemistry editor Austin Paterson, in which it was indicated that the word „density" should be abbreviated in the dictionary by the uppercase letter „D” or by the lowercase letter „d” (Ticak, 2016). At the day of their meeting, Mikhael is late. When he finally makes it, entering the restaurant, he sees that Stephanie is talking with a stranger, at the next table. Surprised to see that, he asks Daniel what is Stephanie talking about with the unknown man. In this context, Daniel utters the following sentence:

(8) Stephanie is referring to a ghost word.

In what follows, I let $S$ denote the speaker and $H$ denote the hearer of the above example. In order to game-theoretically model the aforementioned situation of strategic communication between $S$ and $H$, I will assume that both are rational agents and that they display semantic competence with regard to the fragment of natural language containing sentences consisting of ITVs. Where the function $\mu$ is the semantics of this language fragment, I will assume that the values of $\mu$ are elements from the power set of the set of propositions expressed by the utterances of sentences with ITVs. In case $S$ utters a sentence with the ITV „refer (to)” whose surface syntax is probabilistically silent about the meaning he intends to express, I assume that one of the intensional, de dicto interpretation and the extensional, de re interpretation, is more likely than the other, and that, being a rational agent, $H$ knows that and, in spite of his interpretative uncertainty regarding the intended meaning, he also knows which interpretation is more probable (Parikh, 1991, 2000, 2001, 2010). In the foregoing example, the latter interpretation is more plausible than the other. Also, I assume that the effort of producing or of processing a linguistic form which transparently expresses $S$ 's referential intention is greater than the effort of producing or of processing a linguistic form in which this intention is not syntactically profiled, and that both $S$ and $H$ are cost minimizers (Clark, 2012; Parikh, 1991, 2000, 2001, 2010). Generally, in case $S$ utters a sentence like the aforementioned one, I will assume that the intention of $H$ regarding its interpretation, and the referential intention of $S$, even though the latter is 
attuned to a particular de dicto or de re meaning, are not common knowledge to $S$ and $H$ (Parikh, 1991, 2000, 2001). Finally, I consider that all these above descriptions are common knowledge to both agents.

In what follows, I will show that in the game by means of which the above described scenario is modelled, the assumptions introduced before guarantee the existence of a unique solution concept according to which the optimal choice of the speaker is to use the ambiguous sentence (8) and the optimal choice of the hearer is to assign to (8) an extensional, de re interpretation.

After $S$ utters the sentence (8), $H$ knows that its utterance can receive either an intensional, de dicto interpretation, or an extensional, de re one. In this regard, $H$ knows that on the de dicto interpretation of the utterance, the indefinite NP from (8) is logically governed by the semantic verb „refer (to)" and, taking narrow scope with respect to it, generates a non-specific reading according to which the referent of the subject phrase of (8) is not referring to a particular ghost word. Likewise, $H$ knows that on the de re interpretation of the very same utterance, the intensional NPcomplement which occurs in (8) takes wide scope with respect to the intensional transitive semantic verb and generates the specific reading according to which there is a particular word, namely „dord”, to which the referent of the proper name from (8) is referring to. Insofar as $H$ does not know which proposition $S$ intends to express by uttering the sentence (8), $H$ does not know whether the indefinite NP which occupies the object position of the intensional verb in (8) does call or not for one specific satisfier. Aware of the fact that he has the two aforementioned interpretative choices, $H$ knows that his optimal choice between them depends on which information state of $S$ is factual.

Let $m_{1}$ abbreviate the intensional, de dicto meaning of the utterance of the sentence (8) and let $m_{2}$ abbreviate the extensional, de re meaning of the same utterance. Also, let $s_{1}$ denote the information state of the agent $S$ in which he has the intention to convey, by uttering the sentence (8), the meaning $m_{1}$, and let $s_{2}$ stand for an information state in which, this time, the referential intention of $S$ is to convey, by uttering the very same sentence, the meaning $m_{2}$.

The referential intention of $S$ to communicate to $H$ that the former is in the information state $s_{1}$, can be accomplished in at least two ways. In this sense, $S$ can choose to utter the ambiguous sentence (8) displayed above, or he can choose to utter, in order to transparently signal to $H$ that the factual information state is $s_{1}$, an alternative longer sentence consisting of an expression which semantically induces non-specificity, like the following: 
(9) Stephanie is referring to a ghost word, not a particular one.

Also, the referential intention of $S$ to signal to $H$ the meaning $m_{2}$ can be accomplished in many ways. In this regard, $S$ can choose to utter, as before, the ambiguous sentence (8), or he can choose to utter, in order to clarify that $s_{2}$ is the factual information state, an alternative linguistic expression, like the following:

(10) Stephanie is referring to a ghost word she has recently heard, namely „dord”.

$S$ 's rationality ensures that before uttering a sentence which conveys his intended meaning, $S$ prospects his alternative linguistic choices and assesses their consequences in order to find the optimal action (Clark, 2012; Parikh, 1991, 2000, 2001, 2007, 2010). $S$ knows that the utterance of any of the sentences (9) and (10) aforementioned, in spite of the complexity induced by the supplementary linguistic material, would place $H$ in an information state in which there is no uncertainty with regard to what the former intends to communicate to the latter, and knows also that this fact is common knowledge to both of them. In this regard, let's consider that $t_{1}$ is the information state in which $S$ sends $H$ in case the former would choose to utter the sentence (9), that $t_{2}$ and $t_{3}$ are the information states induced by $S$ 's actual choice to utter the ambiguous sentence (8), and that $t_{4}$ is the information state in which $H$ would be placed in case $S$ would choose to utter the sentence (10).

In the scenario mentioned above, only $s_{2}$ is the factual information state of $S$ and only $S$ can epistemically discern between $s_{1}$ and $s_{2}$. The fact that $H$ lacks information which would allow him to adjudicate which of the information states $s_{1}$ and $s_{2}$ is factual, and that he is in an information state in which, given his epistemic indiscernibility between $t_{2}$ and $t_{3}$, he fails to recognize the actual referential intention of $S$, is common knowledge to both agents (Clark, 2012; Parikh, 1991, 2000, 2001, 2007, 2010). Insofar as the strategic interaction of $S$ and $H$ modelled by the present game evolves in the same way as that of the speaker and the hearer which was described in the first part of this paper, and given that, in the present scenario, the mutual recursive reasonings of $S$ and $H$ about each of the other's choices replicate the agents' cognitive dynamics and the interplay described there, I will skip the details. 
In the previously mentioned example, the agent $H$ is in the epistemic position to infer that the information state $s_{2}$ of the speaker is more likely than the information state $s_{1}$. Let the symbol $p_{1}$ denote the probability that $S$ is in the information state $s_{1}$ in which his referential intention is attuned to the meaning $m_{1}$, and let the symbol $p_{2}$ denote the probability that the information state $s_{2}$, in which $S$ 's referential intention is attuned to the meaning $m_{2}$, is the factual state. Insofar as in the foregoing example the information state $s_{2}$ is more likely than $s_{1}$, and so the value of the probability function $p_{2}$ is greater than the value of $p_{1}$, I will assume, for simplicity, that the value of $p_{2}$ is 0.8 , which means that the value of $p_{1}$, given that axiomatically $p_{1}=1-p_{2}$, is 0.2 . Hence, even though $H$ cannot epistemically discriminate between $t_{2}$ and $t_{3}$, he considers that the probability of being located in the information state $t_{3}$ is four times greater than that of being located in the information state $t_{2}$ and this is common knowledge to $S$ and $H$.

In order to find the solution of the coordination problem raised by the actual choice of $S$ to utter the sentence (8), $H$ has to take into account $S$ 's other possible linguistic choices (9) and (10) and to assess their consequences. In this sense, given the agents' rational tendency of being cost minimizers, their preferences for spending the least effort in the linguistic production and processing work, and their preference for successful communication, are common knowledge to both $S$ and $H$ (Clark, 2012; Parikh, 1991, 2000, 2001, 2010). In this regard, I will assume that the benefit of $S$ and $H$ of converging on the correct interpretation of an utterance equals 30 units and that the penalty, which their miscommunication incurs, values -10 units. Let the symbols $u_{1}, u_{2}$ and $u_{3}$ denote, this time, the utterances of the above sentences (8), (9) and (10). Insofar as the effort of producing an utterance which linguistically articulates the referential intention of the speaker, like $u_{2}$ and $u_{3}$, is greater than the effort of producing the ambiguous utterance $u_{1}$, I will assume that the cost of producing $u_{2}$ or $u_{3}$ is 10 units, while the cost of producing $u_{1}$ is 5 units. Given that the lengths of the sentences is correlated with the effort of processing the corresponding utterances, I will assume that, for both $S$ and $H$, the cost of processing $u_{1}$ is 5 units, while the cost of processing $u_{2}$ or $u_{3}$ is 10 units. All these data are presented in the following table. 
Table 1. Costs and benefits

\begin{tabular}{ccc}
\hline Benefits / Costs & $S$ & $H$ \\
\hline Correct information & +30 & +30 \\
Incorrect information & -10 & -10 \\
Producing and processing $u_{1}$ & -10 & -5 \\
Producing and processing $u_{2}$ or $u_{3}$ & -20 & -10 \\
\hline
\end{tabular}

Insofar as the agents' utilities are determined by the above displayed benefits and costs, both $S$ and $H$ have to factor them into their computation of the optimal action needed for their coordination. As usual, the utilities are assigned to the members of the set of strategic profiles which corresponds to the Cartesian product of the set of the speaker's strategies and the set of the hearer's strategies (Clark, 2012; Parikh, 1991, 2000, 2001, 2007, 2010). Considering that $a_{S}, a_{H}, f_{s}, f_{H}$ are the utilities the agents receive when they successfully communicate with the costlier $u_{2}$ and $u_{3}$, that $b_{S}, b_{H}, e_{S}, e_{H}$ are those obtained when their linguistic coordination involves the least effort, and that $c_{S}, c_{H}, d_{S}, d_{H}$ correspond to the cases of miscommunication, their values are the following:

$$
\begin{aligned}
& a_{S}=f_{S}=10, b_{S}=e_{S}=20, c_{S}=d_{S}=-20 \\
& a_{H}=f_{H}=20, b_{H}=e_{H}=25, c_{H}=d_{H}=-15
\end{aligned}
$$

In order to find the solution concept of the game which models the foregoing example in which $S$ has the referential intention to express the meaning $m_{2}$ and, to this end, chooses to utter an ambiguous sentence, creating thus a semantic decision problem for $H$, we have to compute the expected utilities for each profile of strategies. In what follows, I will use the same notation that I have used in the first part of the paper to present the agents' strategies, but bear in mind that the meanings of the symbols used there in the strategies' notation, are now adjusted to accommodate the data of the aforementioned scenario of strategic interaction between $S$ and $H$. Considering the above probabilistic distribution over the set of the information states of $S$, the vectors below display the expected utilities computed for each of the agents' joint strategies. 


$$
\begin{array}{ll}
\left(\mathrm{P}_{1}\right) \quad\left(s_{1} \rightarrow u_{1}, s_{2} \rightarrow u_{1} ; t_{1} \rightarrow m_{1},\left\{t_{2}, t_{3}\right\} \rightarrow m_{1}, t_{4} \rightarrow m_{2}\right) \\
\\
p_{1}(20,25)+p_{2}(-20,-15)=(4,5)+(-16,-12)=(-12,-7) \\
\left(\mathrm{P}_{2}\right) \quad\left(s_{1} \rightarrow u_{2}, s_{2} \rightarrow u_{1} ; t_{1} \rightarrow m_{1},\left\{t_{2}, t_{3}\right\} \rightarrow m_{1}, t_{4} \rightarrow m_{2}\right) \\
& p_{1}(10,20)+p_{2}(-20,-15)=(2,4)+(-16,-12)=(-14,-8) \\
& \left(s_{1} \rightarrow u_{1}, s_{2} \rightarrow u_{3} ; t_{1} \rightarrow m_{1},\left\{t_{2}, t_{3}\right\} \rightarrow m_{1}, t_{4} \rightarrow m_{2}\right) \\
\left(\mathrm{P}_{3}\right) \quad & p_{1}(20,25)+p_{2}(10,20)=(4,5)+(8,16)=(12,21) \\
& \left(s_{1} \rightarrow u_{2}, s_{2} \rightarrow u_{3} ; t_{1} \rightarrow m_{1},\left\{t_{2}, t_{3}\right\} \rightarrow m_{1}, t_{4} \rightarrow m_{2}\right) \\
\left(\mathrm{P}_{4}\right) \quad & p_{1}(10,20)+p_{2}(10,20)=(2,4)+(8,16)=(10,20) \\
& \left(s_{1} \rightarrow u_{1}, s_{2} \rightarrow u_{1} ; t_{1} \rightarrow m_{1},\left\{t_{2}, t_{3}\right\} \rightarrow m_{2}, t_{4} \rightarrow m_{2}\right) \\
\left(\mathrm{P}_{5}\right) \quad p_{1}(-20,-15)+p_{2}(20,25)=(-4,-3)+(16,20)=(12,17) & \\
& \left(s_{1} \rightarrow u_{2}, s_{2} \rightarrow u_{1} ; t_{1} \rightarrow m_{1},\left\{t_{2}, t_{3}\right\} \rightarrow m_{2}, t_{4} \rightarrow m_{2}\right) \\
\left(\mathrm{P}_{6}\right) \quad & p_{1}(10,20)+p_{2}(20,25)=(2,4)+(16,20)=(18,24) \\
& \left(s_{1} \rightarrow u_{1}, s_{2} \rightarrow u_{3} ; t_{1} \rightarrow m_{1},\left\{t_{2}, t_{3}\right\} \rightarrow m_{2}, t_{4} \rightarrow m_{2}\right) \\
\left(\mathrm{P}_{7}\right) \quad & p_{1}(-20,-15)+p_{2}(10,20)=(-4,-3)+(8,16)=(4,13) \\
& \left(s_{1} \rightarrow u_{2}, s_{2} \rightarrow u_{3} ; t_{1} \rightarrow m_{1},\left\{t_{2}, t_{3}\right\} \rightarrow m_{2}, t_{4} \rightarrow m_{2}\right) \\
& p_{1}(10,20)+p_{2}(10,20)=(2,4)+(8,16)=(10,20)
\end{array}
$$

The computations presented above allow us to verify whether the game of partial information which models the strategic interaction between $S$ and $H$ in the example analysed in this paper, has a solution concept. Such a solution concept would correspond to a Nash equilibrium of the game and would guarantee that the selected strategic profile is an optimal one, given that neither agent is motivated to alter his strategy while the other agent does not deviate from his choice (Clark, 2012; Parikh, 1991, 2000, 2001, 2007, 2010). A quick inspection of the equations corresponding to the computations of the expected utilities, shows that the set of Nash equilibria is not, as desired, a singleton, but contains the following two elements $\left(\mathrm{P}_{3}\right)$ and $\left(\mathrm{P}_{6}\right)$. In this regard, knowing the structure of the game, both $S$ and $H$ commonly know that there are two optimal strategic profiles in which they successfully converge on the interpretation of the utterance $u_{1}$. Hence, the first Nash equilibrium predicts that in the information state $s_{2}, S$ chooses to express the more probable meaning $m_{2}$ by the costlier utterance $u_{3}$ and that, insofar as he chooses to use the ambiguous utterance $u_{1}$ in the information 
state $s_{1}, H$ chooses to assign to $u_{1}$ its de dicto interpretation $m_{1}$. Likewise, according to the strategic profile corresponding to the second Nash equilibrium of this game, $S$ decides to use the unambiguous utterance $u_{2}$ in the information state $s_{1}$, and given that his factual information state is $s_{2}$, he chooses to utter the ambiguous utterance $u_{1}$, while $H$ chooses to assign to $u_{1}$ its de re interpretation $m_{2}$. In spite of knowing all the above conditions in which the agents' coordination on an interpretation of $u_{1}$ occurs, $H$ does not know yet what his optimal choice is and so, he remains totally undecided regarding whether the correct interpretation he should assign to $u_{1}$, the one which would accurately reproduce $S$ 's referential intention, is $m_{1}$ or $m_{2}$.

However, the game of partial information which models our example of a particular situation in which the ambiguity is present in the communication act due to the intensionality of the semantic verb ,refer (to)", has a unique solution concept. In order to find it, we have to sweep away from the set of Nash equilibria those elements which violate the condition of being Pareto efficient (Clark, 2012; Parikh, 1991, 2000, 2001, $2007,2010)$. In the present case, insofar as the strategic profile $\left(\mathrm{P}_{6}\right)$ yields a higher expected utility than the other strategic profile $\left(\mathrm{P}_{3}\right)$, and given that it is common knowledge to both agents that their rationality requires them to maximize the expected utility, $S$ and $H$ choose to play only those strategies which orient them towards the Nash equilibrium $\left(\mathrm{P}_{6}\right)$ which passes through the Paretian filter. Therefore, the unique solution concept of the game by means of which I represented above a particular linguistic interaction in which the emblematic semantic verb ,refer (to)" is involved, predicts that $S$ chooses to express the more probable meaning $m_{2}$ by means of the ambiguous utterance $u_{1}$, that he chooses to express the least probable meaning $m_{1}$ by means of the unambiguous utterance $u_{2}$, and that $H$ chooses to assign to the utterance $u_{1}$, in the aforementioned scenario, the extensional, de re interpretation $m_{2}$ according to which the referent of the subject phrase in (8) is referring to the particular ghost word ,dord”.

What would happen in case the aforementioned context would offer clues indicating to $H$ that the most probable meaning of the ambiguous utterance $u_{1}$ is its intensional, de dicto interpretation $m_{1}$ corresponding to the non-specific reading of the sentence (8)? In this hypothetical case, the above probabilistic distribution over the information states $s_{1}$ and $s_{2}$ will be modified by the supplementary contextual information, so that now the probability that $s_{1}$ is the factual information state of $S$ will be greater than the probability of $s_{2}$, and that would be common knowledge to both $S$ and $H$. In this case, as we have seen in the first part of the present paper, insofar as the 
agents' foregoing utilities satisfy, as it happens in our scenario, the following conditions

$$
\begin{aligned}
& b_{S}-a_{S}=e_{S}-f_{S} \\
& b_{H}-a_{H}=e_{H}-f_{H}
\end{aligned}
$$

the latter equations ensure that the unique Pareto-Nash equilibrium will correspond, this time, not to the strategic profile $\left(\mathrm{P}_{6}\right)$, but to the strategic profile $\left(\mathrm{P}_{3}\right)$ instead.

In order to confirm and to transparentize this game-theoretical prediction, I will modify the initial probabilistic distribution over the set of the information states of $S$ and I will display below the computations of the expected utilities of the agents' joint strategies for the following specific values $p_{1}=0.7, p_{2}=1-p_{1}=0.3$.

$$
\begin{array}{ll}
\left(\mathrm{P}_{1}\right) \quad\left(s_{1} \rightarrow u_{1}, s_{2} \rightarrow u_{1} ; t_{1} \rightarrow m_{1},\left\{t_{2}, t_{3}\right\} \rightarrow m_{1}, t_{4} \rightarrow m_{2}\right) \\
& p_{1}(20,25)+p_{2}(-20,-15)=(14,17.5)+(-6,-4.5)=(8,13) \\
& \left(\mathrm{P}_{2} \rightarrow u_{2}, s_{2} \rightarrow u_{1} ; t_{1} \rightarrow m_{1},\left\{t_{2}, t_{3}\right\} \rightarrow m_{1}, t_{4} \rightarrow m_{2}\right) \\
& p_{1}(10,20)+p_{2}(-20,-15)=(7,14)+(-6,-4.5)=(1,9.5) \\
& \left(\mathrm{P}_{1} \rightarrow u_{1}, s_{2} \rightarrow u_{3} ; t_{1} \rightarrow m_{1},\left\{t_{2}, t_{3}\right\} \rightarrow m_{1}, t_{4} \rightarrow m_{2}\right) \\
& p_{1}(20,25)+p_{2}(10,20)=(14,17.5)+(3,6)=(17,23.5) \\
& \left(\mathrm{P}_{1} \rightarrow u_{2}, s_{2} \rightarrow u_{3} ; t_{1} \rightarrow m_{1},\left\{t_{2}, t_{3}\right\} \rightarrow m_{1}, t_{4} \rightarrow m_{2}\right) \\
& p_{1}(10,20)+p_{2}(10,20)=(7,14)+(3,6)=(10,20) \\
& \left(\mathrm{P}_{4} \rightarrow u_{1}, s_{2} \rightarrow u_{1} ; t_{1} \rightarrow m_{1},\left\{t_{2}, t_{3}\right\} \rightarrow m_{2}, t_{4} \rightarrow m_{2}\right) \\
\left(\mathrm{P}_{5}\right) \quad & p_{1}(-20,-15)+p_{2}(20,25)=(-14,-10.5)+(6,7.5)=(-8,-3) \\
& \left(s_{1} \rightarrow u_{2}, s_{2} \rightarrow u_{1} ; t_{1} \rightarrow m_{1},\left\{t_{2}, t_{3}\right\} \rightarrow m_{2}, t_{4} \rightarrow m_{2}\right) \\
\left(\mathrm{P}_{6}\right) \quad & p_{1}(10,20)+p_{2}(20,25)=(7,14)+(6,7.5)=(13,21.5) \\
& \left(s_{1} \rightarrow u_{1}, s_{2} \rightarrow u_{3} ; t_{1} \rightarrow m_{1},\left\{t_{2}, t_{3}\right\} \rightarrow m_{2}, t_{4} \rightarrow m_{2}\right) \\
& p_{1}(-20,-15)+p_{2}(10,20)=(-14,-10.5)+(3,6)=(-11,-4.5) \\
& \left(\mathrm{P}_{7} \rightarrow u_{2}, s_{2} \rightarrow u_{3} ; t_{1} \rightarrow m_{1},\left\{t_{2}, t_{3}\right\} \rightarrow m_{2}, t_{4} \rightarrow m_{2}\right) \\
& \left.\mathrm{P}_{8}\right) \quad(10,20)+p_{2}(10,20)=(7,14)+(3,6)=(10,20) \\
& p_{1}(10)
\end{array}
$$




\section{Speaker's Reference and Semantic Reference}

In the above situation of communication which shows how the intensionality of the emblematic semantic verb „refer (to)" generates ambiguity at the interpretative level, and which was analysed from a gametheoretical perspective, the focus was on what is usually called the speaker's reference. But besides occurring in sentences used to report the speaker's reference, the intensional verb from our semantic lexicon also occurs in statements of semantic theorizing by means of which we define the references of a language's terms. This latter form of reference, which occurs when the semantic verb is used in a more technical way and only at the metalanguage level of a language, is usually called semantic reference (Abbott, 2010; D'Ambrosio, 2017; Kripke, 1977). In this section, I will present some methodological consequences of framing the understanding of reference by means of the conceptual tools offered by the games of partial information and I will show how the interplay between the speaker's reference and the semantic reference is accommodated in this game-theoretical framework.

The above game of partial information by means of which I represented the agents's coordination on the speaker's reference is, in the whole economy of their strategic interaction, only a local game (Parikh, 1991, 2000, 2001, 2010). Its structure is common knowledge to both $S$ and $H$ only after $S$ linguistically materializes his choice to utter $u_{1}$. Let $G_{L}\left(u_{1}\right)$ abbreviate this game generated by $S$ 's decision act of uttering $u_{1}$. In order to understand how the game of partial information $G_{L}\left(u_{1}\right)$ is embedded in another larger game $G_{G}\left(m_{2}\right)$, let's reflect again about the agents' strategic interaction in our aforementioned scenario in which $S$ utters $u_{1}$ in the information state $s_{2}$ and $H$ interprets the ambiguous utterance $u_{1}$ as meaning $m_{2}$.

$S$ 's decision problem in the game $G_{L}\left(u_{1}\right)$ is to optimally choose an element from the set of his linguistic forms in order to successfully translate his referential intention at the level of the utterance. Hence, identifying the set of the linguistic forms which an agent might choose to utter with the set of his possible utterances, $S$ 's problem is to choose that utterance which ensures the success of his coordination with $H$ on the meaning the former intends to convey. In contrast, $H$ 's decision problem in $G_{L}\left(u_{1}\right)$ is to optimally choose an element from the set of his interpretations which perfectly correlates with $S$ 's referential intention after the latter utters the linguistic form he has chosen. Both agents coordinate, by finding the solution concept of the game $G_{L}\left(u_{1}\right)$, only when each of them is capable to solve in a rationally flawless way the decision problem with which is confronted. But before solving his own decision problem, $S$ has to consider his partner's decision 
problem and to prospect whether $H$ has in the game $G_{L}\left(u_{1}\right)$ the necessary informational resources to find the solution to his problem (Parikh, 1991, $2000,2001,2010)$. Insofar as the structure of the game $G_{L}\left(u_{1}\right)$ is common knowledge to $S$ and $H, S$ knows which of the strategic profiles $\left(\mathrm{P}_{3}\right)$ and $\left(\mathrm{P}_{6}\right)$ is the Nash equilibrium towards which the choice of $H$ will converge. Hence, $S$ knows that $H$ will choose the Pareto efficient Nash equilibrium $\left(\mathrm{P}_{6}\right)$ and that, as a consequence of this choice, $S$ 's utility will be 20 , while $H$ 's utility will be 25 . In this sense, the payoff vector $(20,25)$ is the value of the game $G_{L}\left(u_{1}\right)$ and $S$ knows that. Each element from the set of the linguistic forms which $S$ might have chosen to utter as an alternative to his actual utterance $u_{1}$, that is, each element from $S$ 's set of possible utterances, is associated with a local game of partial information. S's rationality obliges him to choose from his set of possible utterances available to express his referential intention that element $u$ which ensures that the value of the game $G_{L}(u)$ corresponding to $u$ is maximized (Parikh, 1991, 2000, 2001, 2010). Insofar as the utterances $u_{1}$ and $u_{3}$ can both serve to express $S$ 's referential intention, and as the value of the game $G_{L}\left(u_{3}\right)$, defined by the the payoff vector $(10,20)$, is lower than the value of $G_{L}\left(u_{1}\right), S$ 's rationality requires him to choose to play with $H$ the game $G_{L}\left(u_{1}\right)$, and so to utter $u_{1}$.

All the local games of partial information, like $G_{L}\left(u_{1}\right)$ and $G_{L}\left(u_{3}\right)$ above, whose values $S$ has to determine before choosing to perform $u_{1}$, are parts of a more complex game-theoretical structure $G_{G}\left(m_{2}\right)$ which constitutes a global game of partial information (Parikh, 1991, 2000, 2001, 2010). In this regard, $G_{G}\left(m_{2}\right)$ is the game which only $S$ has to optimally solve, by comparing all the local games of partial information, from $G_{G}\left(m_{2}\right)$, which are associated with utterances expressing $m_{2}$, and by choosing that one which pays the most, in order to decide that his best move is, globally, to play the game $G_{L}\left(u_{1}\right)$, and to initiate it, by his act of uttering $u_{1}$.

This more complete game-theoretical way of framing the agents' strategic interaction, presented in (Parikh, 1991, 2000, 2001, 2007, 2010), has important methodological implications for the way in which the complex relation between the speaker's reference and the semantic reference has to be theoretically conceived. As solution to a game of coordination played in the past at a global scale in a society, a selected system of linguistic conventions stating the semantic connections between words and references constitutes a Nash equilibria. In this regard, the linguistic conventions which define words' semantic references emerged on the background of the regular interactions between rational agents. At the point at which an assignment of a semantic reference to a word became operative in a community, embodying a linguistic convention, it automatically reinforced other 
assignments of semantic references and so gave birth to new linguistic conventions. In order to better understand, consider one simple hypothetical situation holding in a society before a network of semantic reference relations being effectual there. In this pre-play state, presented in (Parikh, 2007, 2010), there are two semantic values $m_{i}$ and $m_{j}$, and two linguistic forms $u_{i}$ and $u_{j}$. At this stage, any of the linguistic forms $u_{i}$ and $u_{j}$ can be used for any of the semantic values $m_{i}$ and $m_{j}$. In case the linguistic form $u_{i}$ is selected for the semantic value $m_{i}$, the other linguistic form $u_{j}$ will be simultaneously reserved for the semantic value $m_{j}$, and vice-versa. There are at least two Nash equilibria in this game played across the entire society and they correspond to two systems of semantic connections that can emerge: either $u_{i}$ is selected for $m_{i}$ and $u_{j}$ is chosen for $m_{j}$, or $u_{i}$ is reserved for $m_{j}$ and $u_{j}$ is selected for $m_{i}$. Suppose now that one of these equilibria is selected by the community, let's say the first one. Once the choice of $u_{i}$ for expressing $m_{i}$ is operative, by default $u_{j}$ will not be available anymore for expressing $m_{i}$, and equivalently, reserving $u_{j}$ for the meaning $m_{p}$, simultaneously rules out the case of $u_{i}$ being available for expressing $m_{j}$. So, the equilibrium selected as solution to the above game gives rise to a system: the two semantic reference relations, and their corresponding linguistic conventions, one stating that $m_{i}$ is the semantic reference of $u_{i}$ and the other stating that $m_{j}$ is the semantic reference of $u_{j}$, evolve simultaneously and reinforce one another (Parikh, 2007, 2010: 295).

Hence, once a linguistic form receives a semantic reference, the relation holding between the former and the latter is conditioned by the relation which the other linguistic forms have with their corresponding semantic references (Parikh, 2010: 293). This fact of words systemically and simultaneously receiving, in an equilibrium state, their semantic references, impacts the way in which they conventionally connect with the world, and $a$ fortiori, the way in which they are used, in speakers' references, to talk about the world. In this regard, the relation underlying a speaker's reference to something in the world is mediated by the relations underlying the words' semantic references. This shows that the way in which a descriptive, a pictorial, a functional or a causal theory of reference conceptually frames the relation between the speaker's reference and the semantic reference, stating that the latter depends on the former (D'Ambrosio, 2017), does not offer a complete and accurate picture of their relationship. In this sense, disregarding the fact that the speaker's reference is also anchored in the relation of semantic reference, all these accounts do not capture their mutual dependence and miss the point according to which the relation between the speaker's reference and the semantic reference constitutes, in a game- 
theoretical manner of speaking, an equilibrium of fixed point (Parikh, 2010: 293). In this sense, insofar as the system of the semantic reference relations emerging in a society constitutes a Nash equilibrium semantic reference, and as the game of partial information, presented in this paper, results in an equilibrium speaker's reference, according to which the hearer succeeds in coordinating with the speaker on the latter's intended meaning, grasping his referential intention, these two equilibria interact and give rise to an equilibrium between the equilibria (Parikh, 2010: 295).

A first point of intersection between the semantic reference and the speaker's reference occurs at the level of the global game of partial information, like the game $G_{G}\left(m_{2}\right)$ above, which the speaker $S$ has to solve in order to decide to play a speaker's reference game, like the reference game $G_{L}\left(u_{1}\right)$ above. In this regard, when $S$, in order to coordinate with $H$ on the meaning he intends to convey, considers the set of linguistic forms apt to express his referential intention and selects from it the most fitted one, $S$ exploits his knowledge of the system of semantic reference relations. This knowledge of the linguistic conventions which state the semantic references relations underlies $S$ 's ability to inspect, before choosing to play the reference game with the highest value, such as $G_{L}\left(\mathcal{U}_{1}\right)$ above, the local games of partial information corresponding to all of his linguistic choices available to express his referential intention.

As seen above, in a local game of partial information, like the aforementioned game between $S$ and $H$ which served to model the communication involving the paradigmatic semantic verb ,refer (to)", the arena of the semantic references ensures the success of the speaker's reference. A second entry point of the semantic reference in a game of partial information, like $G_{L}\left(u_{1}\right)$ above, is transparent in the way in which the hearer $H$ manages to find the utterance's interpretation corresponding to the speaker's reference. Insofar as the knowledge of the system of semantic reference relations also mediates the hearer's comprehension of the speaker's reference, and therefore conditions his coordination with $S$, the arena of the semantic references is not reserved only to the player which initiates the game of partial information. Insofar as $H$ 's knowledge of the semantic reference relations underlies his ability to prospect the linguistic alternatives $S$ might have chosen to utter in order to convey his referential intention, it can be said that both agents walk on the same Nash stable semantic reference ground in their search for coordination on the speaker's reference. In this regard, a story as the one Abbott tells in (Abbott, 2010), according to which the speaker's reference and the semantic reference distribute across the roles played by the agents involved in communication, 
is not complete. According to Abbott, while the speaker's reference obviously belongs to the side of the „language producer", the semantic reference stands on the side of the "language consumer" because, in his understanding of the speaker's reference, the hearer $H$ relies on the knowledge of the semantic reference relations encoded by the linguistic conventions (Abbott, 2010: 275). But, as it was seen above, in the aforementioned game of partial information $G_{L}\left(u_{1}\right)$, the mutually recursive inferences of the players and their cognitive dynamics ensure that, in order to coordinate on the speaker's reference, each agent rationally puts himself in the shoes of the other agent. Far for being static, the roles of the agents in the communication scheme reverse in their mutual reasoning about the other's optimal choice, and the higher-order reasoning processes, involved in both the production of an utterance and in its interpretation, exploit the knowledge of the semantic reference relation. Also, the fact that the system of semantic reference relations is known and exploited by both the speaker and the hearer caught in a strategic interaction situation, was already transparent at the level of the assumption of the common knowledge of their semantic competence, assumption which was operative in the above game $G_{L}\left(u_{1}\right)$.

As it was seen above, the key verb „refer (to)”, which is central to our semantic lexicon, being an ITV, as D'Ambrosio showed in (D'Ambrosio, 2017), is structurally ambiguous between an intensional reading which conveys a de dicto reference and an extensional reading which conveys a de re reference. Moreover, in his excellent paper, D'Ambrosio shows that the semantic reference inherits both readings of the emblematic semantic verb used to report the speaker's reference, and that, in consequence, the statements used in our semantic theorizing in which this verb occurs are also structurally ambiguous between a de dicto semantic reference and a de re semantic reference (D'Ambrosio, 2017). According to D'Ambrosio, in case it is considered that the verb occurring in the following semantic postulate

(11) „Hesperus” refers to Hesperus.

has a de re semantic reference, (11) receives an interpretation which entails that the sentence

(12) „Hesperus” refers to Phosphorus. 
is true. In contrast, considering that the intensional verb which occurs in (11) has a de dicto semantic reference, the entailment of the latter sentence from the first sentence is not a logically valid manoeuvre.

The ambiguity inherent to the linguistic conventions corresponding to the semantic reference relations makes their efficiency transparent. A language is efficient by virtue of the fact that the very same linguistic material can be refashioned and adjusted to contextually express a variety of semantic values (Parikh, 2010: 22). Insofar as the natural languages' pervasive ambiguity displays their efficiency, and as the linguistic conventions are formulated with the tools of these languages, the linguistic conventions governing the agents' coordination in strategic communication situations, will import their efficiency from the languages in which they are stated. Moreover, the efficiency of the linguistic conventions due to their ambiguity shows, once again, the ineliminability of the contextual information from any theoretically robust account of the production and the comprehension of the speaker's reference. In this regard, the gametheoretical conceptual framework presented in this paper is, as it was seen above, apt to efficiently accommodate the language's efficiency and the key role which the context plays in the interplay between the speaker's reference and the semantic reference.

\section{Conclusions}

I have focused, in this paper, on the most emblematic verb of the semantic idiom and I have presented the theoretical and the empirical grounds favouring the view that it displays the semantic behaviour of a typical ITV. Also, I have presented a scenario of communication with an utterance of a sentence formed with the standard semantic verb, and I have represented it as a two-agent game of partial information. In this regard, in order to show how the semantic verb's inherent ambiguity, between the intensional, de dicto and the extensional, de re interpretation, is rationally solved in the game-theoretical conceptual framework adopted in this paper, I have computed the expected utilities of the joint strategies, and I have determined the game's unique solution concept in the form of a Nash equilibrium which satisfies the condition of Pareto efficiency. In the end, I have presented some of the methodological consequences of framing the understanding of reference by means of the conceptual tools offered by the games of partial information, and I have shown how the complex interplay between the speaker's reference and the semantic reference is accommodated in this game-theoretical framework. 


\section{References}

Abbott, B. (2010). Reference. Oxford, UK: Oxford University Press.

Almog, J. (1998). The subject verb object class I. Philosophical Perspectives, 12(S12), 39-76. doi:10.1111/0029-4624.32.s12.2

Clark, R. (2012). Meaningful games. Exploring language with game theory. Cambridge, USA: MIT Press.

D’Ambrosio, J. (2017). Semantic verbs are intensional transitives. Mind (2017, December 21). doi:10.1093/mind/fzx029. Retrieved from http://justindambrosio.wixsite.com/philosophy/research

Forbes, G. (2013). Intensional transitive verbs. In E. N. Zalta (Ed.), The Stanford encyclopedia of philosophy. Stanford, USA: Stanford University. Retrieved from https://plato.stanford.edu/archives/fall2013/entries/intensional-transverbs

Kripke, S. (1977). Speaker's reference and semantic reference. Midwest Studies in Philosophy, 2(1), 255-276. doi:10.1111/j.1475-4975.1977.tb00045.x

Lappin, S. (2017). Formal semantics. In M. Aronoff \& J. Ress-Miller (Eds.), The bandbook of linguistics (2nd ed.) (pp. 279-298). London, UK: Wiley-Blackwell.

Moltmann, F. (1997). Intensional verbs and quantifiers. Natural Language Semantics, 5(1), 1-52. doi:10.1023/a:1008245409172

Parikh, P. (1991). Communication and strategic inference. Linguistics and Philosophy, 14(5), 473-514. doi:10.1007/bf00632595

Parikh, P. (2000). Communication, meaning, and interpretation. Linguistics and Philosophy, 23(2), 185-212. doi:10.1023/a:1005513919392

Parikh, P. (2001). The use of language. Stanford, USA: CSLI Publications.

Parikh, P. (2007). Situations, rules, and conventional meaning: Some uses of games of partial information. Journal of Pragmatics, 39(5), 917-933.

doi:10.1016/j.pragma.2007.01.004

Parikh, P. (2010). Language and equilibrium. Cambridge, UK: MIT Press.

Recanati, F. (2000). Oratio obliqua, oratio recta: An essay on metarepresentation. Cambridge, UK: MIT Press.

Richard, M. (2013). Context and the attitudes: meaning in context. (vol. 1). Oxford, UK: Oxford University Press.

Sainsbury, M. (2018). Thinking about things. Oxford, UK: Oxford University Press.

Ticak, M. (2016). What are ghost words?. Grammarly Blog. Retrieved from https://www.grammarly.com/blog/ghost-words /

Zimmermann, T. E. (1993). On the proper treatment of opacity in certain verbs. Natural Language Semantics, 1(2), 149-179. doi:10.1007/bf00372561 
Intensionality, Reference and Games of Partial Information II Mihai HÎNCU

Zimmermann, T. E. (2006). Monotonicity in opaque verbs. Linguistics and Pbilosophy, 29(6), 715-761. doi:10.1007/s10988-006-9009-z 